\title{
Periodic state-space representations of periodic convolutional codes
}

\author{
Diego Napp - Ricardo Pereira - Raquel \\ Pinto · Paula Rocha
}

the date of receipt and acceptance should be inserted later

\begin{abstract}
In this paper we study the representation of periodically time-varying convolutional codes by means of periodic input-state-output models. In particular, we focus on period two and investigate under which conditions a given two-periodic convolutional code (obtained by alternating two time-invariant encoders) can be represented by a periodic input-state-output system. We first show that one cannot expect, in general, to obtain a periodic input-state-output representation of a periodic convolutional code by means of the individual realizations of each of the associated time-invariant codes. We, however, provide sufficient conditions for this to hold in terms of the column degrees of the associated column reduced generator matrices. Moreover, we derive a sufficient condition to obtain a periodic statespace realization that is minimal. Finally, examples to illustrate the results are presented.
\end{abstract}

Keywords Convolutional codes · Periodically time-varying codes · Input-stateoutput representations

\section{Introduction}

Convolutional codes [8] are an important type of error correcting codes that can be represented as a time-invariant discrete linear system over a finite field [16]. They

This work was supported in part by the Portuguese Foundation for Science and Technology (FCT-Fundação para a Ciência e a Tecnologia), through CIDMA - Center for Research and Development in Mathematics and Applications, within project UID/MAT/04106/2013 and also by Project POCI-01-0145-FEDER-006933 - SYSTEC - Research Center for Systems and Technologies - funded by FEDER funds through COMPETE2020 - Programa Operacional Competitividade e Internacionalização (POCI) - and by national funds through FCT Fundação para a Ciência e a Tecnologia.

CIDMA - Center for Research and Development in Mathematics and Applications, Department of Mathematics, University of Aveiro, Aveiro, Portugal E-mail: diego@ua.pt · CIDMA - Center for Research and Development in Mathematics and Applications, Department of Mathematics, University of Aveiro, Aveiro, Portugal E-mail: ricardopereira@ua.pt · CIDMA - Center for Research and Development in Mathematics and Applications, Department of Mathematics, University of Aveiro, Aveiro, Portugal E-mail: raquel@ua.pt · SYSTEC, Faculty of Engineering, University of Porto, Portugal E-mail: mprocha@fe.up.pt 
are used to achieve reliable data transfer, for instance, in mobile communications, digital video and satellite communications $[8,19]$.

Since the sixties it has been widely known that convolutional codes and linear systems defined over a finite field are essentially the same objects, see for instance [16] and references therein. More recently, there has been a new and increased interest in this connection and many advances have been derived from using the system theoretical framework, and in particular of input-state-output representations when dealing with convolutional codes, see $[10,14]$. Most of the large body of literature on convolutional codes and on the relation of these codes with linear systems has been devoted to the "time-invariant" case.

In this work we aim at studying time-varying convolutional codes from a system theoretical point of view. These codes have attracted much attention after Costello conjectured in [4] that nonsystematic time-varying convolutional codes can attain larger free distance than the nonsystematic time-invariant ones. Since then, several researchers have investigated such codes $[3,12,13,15]$. Moreover, in combination with wavelets [5] time-varying convolutional codes yield unique trellis structures that resulted in fast and low computational complexity decoding algorithms.

On the other hand, the theory of periodic input-state-output linear systems has been also an active area of research $[2,11]$ and many interesting results have been obtained in this context. However, little is known on the relation of time-varying convolutional codes and time-varying input-state-output linear systems.

Here we aim at studying this relation and in particular to investigate how to build periodic input-state-output representations for given periodically time-varying convolutional codes (for short, periodic codes). We first observe, and illustrate by means of an example, that the periodic input-state-output representation obtained from the realization of each of the convolutional encoders that define the periodic code, does not necessarily correspond to the original periodic encoding map. We show, however, that if such encoders possess the same column degrees, then the former periodic realization does correspond to the same periodic encoding map. Moreover, in the case of different columns degrees, we still provide a method to obtain a periodic input-state-output representation for a given periodic code. A sufficient condition for obtaining minimality in the realization is also provided.

\section{Preliminaries}

In the sequel we shall follow the system theory notation and consider column vectors rather than row vectors.

\subsection{Time-invariant convolutional codes}

Let $\mathbb{F}$ be a finite field and let $\mathbb{F}[z]$ be the polynomial ring. In a module theoretic point of view, we define a convolutional code as follows. 
Definition 1 Let $\mathbb{F}$ be a finite field and $n, k$ be positive integers with $k<n$. A time-invariant convolutional code $\mathcal{C}$ of rate $k / n$ is a submodule $\mathbb{F}^{n}[z]$ described as

$$
\mathcal{C}=\left\{v(z) \in \mathbb{F}^{n}[z]: v(z)=G(z) u(z), u(z) \in \mathbb{F}^{k}[z]\right\}
$$

where $G(z) \in \mathbb{F}^{n \times k}[z]$ is a full column rank $n \times k$ polynomial matrix over $\mathbb{F}$, called the encoder, $u(z)$ taking values in $\mathbb{F}^{k}[z]$ is the information vector and $v(z)$ is the codeword.

The encoders of a code $\mathcal{C}$ are not unique; however they only differ by right multiplication by unimodular matrices over $\mathbb{F}[z]$. An encoder $G(z)$ is called column reduced if the sum of its column degrees attains the minimal possible value among all the encoders of the same code. If $G(z) \in \mathbb{F}^{n \times k}[z]$ has column degrees $\nu_{1}, \ldots, \nu_{k}$, it can be written as

$$
G(z)=G_{\mathrm{hc}}\left[\begin{array}{llll}
z^{\nu_{1}} & & & \\
& z^{\nu_{2}} & & \\
& & \ddots & \\
& & & z^{\nu_{k}}
\end{array}\right]+G_{\mathrm{rem}}(z)
$$

where $G_{\text {rem }}(z)$ is a polynomial matrix such that the degree of column $i$ is less than $\nu_{i}, i=1, \ldots, k$, and $G_{\mathrm{hc}} \in \mathbb{F}^{n \times k}$ is a matrix whose $i$-th column contains the coefficients of $z^{\nu_{i}}$ in the $i$-th column of $G(z) . G_{\mathrm{hc}}$ is called the leading column coefficient matrix and $G(z)$ is column reduced if and only if $G_{\mathrm{hc}}$ is full column rank.

We define the degree $\delta$ of a convolutional code as the sum of the column degrees of one, and hence any, column reduced encoder. Note that the list of column degrees (also known as Forney indices) of a column reduced encoder is unique up to a permutation. A code $\mathcal{C}$ of rate $k / n$ and degree $\delta$ is said to be an $(n, k, \delta)$ code.

\subsection{Periodically time-varying convolutional codes}

In this work we consider convolutional $\operatorname{codes} \mathcal{C}$ with 2 -periodic encoders. Next we introduce the definition of such encoders (or encoding maps) together with the definition of the corresponding 2-periodic (time-varying) convolutional codes, see $[4,15,18]$. For the purposes of the paper, we present it in a polynomial fashion.

Definition 2 Given two polynomial matrices $G^{0}(z), G^{1}(z) \in \mathbb{F}^{n \times k}[z]$, the periodic encoding map induced by $G^{0}$ and $G^{1}$ is defined as

$$
\begin{aligned}
\phi_{G^{0} G^{1}}: \mathbb{F}^{k}[z] & \longrightarrow \mathbb{F}^{n}[z] \\
u(z) & \longmapsto v(z)
\end{aligned}
$$

where $v(z)=\sum_{i=0}^{+\infty} v_{i} z^{i}$ and $v_{2 \ell+t}=\left(G^{t}(z) u(z)\right)_{2 \ell+t}, t=0,1, \ell \in \mathbb{N}_{0}$, and, moreover, $\left(G^{t}(z) u(z)\right)_{2 \ell+t}$ represents the $(2 \ell+t)$-coefficient of the polynomial $G^{t}(z) u(z)$. 
The corresponding periodic convolutional code $\mathcal{C}_{p}$ is

$$
\begin{gathered}
\mathcal{C}_{p}=\left\{v(z) \in \mathbb{F}^{n}[z]: \exists u(z) \in \mathbb{F}^{k}[z] \text { s.t. (1) holds }\right\} \\
v(z)=\phi_{G^{0} G^{1}}(u(z)) .
\end{gathered}
$$

Such codes will be called 2-periodic convolutional codes.

Inspired by the ideas developed in [2] and [11] we consider the linear map

$$
L_{2}: \mathbb{F}^{n}(z) \rightarrow \mathbb{F}^{2 n}(z)
$$

defined by

$$
L_{2}(v(z))=v^{L}(z), \text { where }\left(v^{L}(z)\right)_{\ell}=\left(\left[\begin{array}{c}
I_{n} \\
z^{-1} I_{n}
\end{array}\right] v(z)\right)_{2 \ell}
$$

and we associate with $\mathcal{C}_{p}$ a time-invariant convolutional code $\mathcal{C}^{L}$, the lifted version of $\mathcal{C}_{p}$, defined as

$$
\mathcal{C}^{L}=\left\{\widetilde{v} \in \mathbb{F}^{2 n}(z): \widetilde{v}=L_{2} v, v \in \mathcal{C}_{p}\right\} .
$$

Note that, since

$$
\left(G^{t}(z) u(z)\right)_{2 \ell+t}=\left(z^{-t} G^{t}(z) u(z)\right)_{2 \ell},
$$

the equation (1) can also be written as

$$
\left(\left[\begin{array}{c}
I_{n} \\
z^{-1} I_{n}
\end{array}\right] v(z)\right)_{2 \ell}=\left(\left[\begin{array}{c}
G^{0}(z) \\
z^{-1} G^{1}(z)
\end{array}\right] u(z)\right)_{2 \ell}, \ell \in \mathbb{N}_{0} .
$$

Moreover, it is possible to make the decomposition

$$
\left[\begin{array}{c}
G^{0}(z) \\
z^{-1} G^{1}(z)
\end{array}\right]=G^{L}\left(z^{2}\right)\left[\begin{array}{c}
I_{k} \\
z^{-1} I_{k}
\end{array}\right]
$$

where

$$
G^{L}(z)=\left[G^{L_{0}}(z) \mid G^{L_{1}}(z)\right]
$$

and the blocks $G^{L_{t}}(z)$ have size $2 n \times k, t=0,1$, and are given by

$$
\begin{aligned}
G^{L_{0}}(z) & =\sum_{i \in \mathbb{N}_{0}}\left[\begin{array}{c}
G_{2 i}^{0} \\
G_{2 i+1}^{1}
\end{array}\right] z^{i}, \\
G^{L_{1}}(z) & =\sum_{i \in \mathbb{N}_{0}}\left[\begin{array}{c}
G_{2 i-1}^{0} \\
G_{2 i}^{1}
\end{array}\right] z^{i}, \text { with } G_{-1}^{0}=0,
\end{aligned}
$$

where the $G_{j}^{t}$ 's are obtained as the coefficients of $G^{t}$, i.e., $G^{t}(z)=\sum_{j \in \mathbb{N}_{0}} G_{j}^{t} z^{j}$.

Thus, the lifted code can be represented as

$$
\mathcal{C}^{L}=\left\{\widetilde{v}(z): \widetilde{v}(z)=G^{L}(z) \widetilde{u}(z), \tilde{u}(z) \in \mathbb{F}^{2 k}[z]\right\},
$$

where $\widetilde{v}=L_{2} v$ and $\widetilde{u}=L_{2} u$.

In the following example we illustrate how to construct the matrix $G^{L}(z)$ given the matrices $G^{0}(z)$ and $G^{1}(z)$. 
Example 1 Considere the field $\mathbb{F}_{2}$ and let

$$
G^{0}(z)=\left[\begin{array}{cc}
z^{2}-z & 1 \\
z^{3} & z \\
z+1 & z^{2}
\end{array}\right] \text { and } G^{1}(z)=\left[\begin{array}{cc}
1-z & z^{3}-z \\
z^{2} & z-z^{2} \\
z^{4}+z & 1
\end{array}\right]
$$

Applying the previous procedure we have that

$$
\left[\begin{array}{c}
G^{0}(z) \\
z^{-1} G^{1}(z)
\end{array}\right]=\left[\begin{array}{cc}
z^{2}-z & 1 \\
z^{3} & z \\
z+1 & z^{2} \\
z^{-1}-1 & z^{2}-1 \\
z & 1-z \\
z^{3}+1 & z^{-1}
\end{array}\right]=\left[\begin{array}{cc}
z^{2} & 1 \\
0 & 0 \\
1 & z^{2} \\
-1 & z^{2}-1 \\
0 & 1 \\
1 & 0
\end{array}\right]+\left[\begin{array}{cc}
-z^{2} & 0 \\
z^{4} & z^{2} \\
z^{2} & 0 \\
1 & 0 \\
z^{2} & -z^{2} \\
z^{4} & 1
\end{array}\right] z^{-1}
$$

and so

$$
G^{L}(z)=\left[\begin{array}{cccc}
z & 1 & -z & 0 \\
0 & 0 & z^{2} & z \\
1 & z & z & 0 \\
-1 & z-1 & 1 & 0 \\
0 & 1 & z & -z \\
1 & 0 & z^{2} & 1
\end{array}\right]
$$

\subsection{State-space realizations}

In systems theory, input-state-output models are mainly used to describe the time evolution of the system signals, which, in the discrete-time case, are time sequences.

Therefore, in the sequel, we sometimes identify an element $a(z)=\sum_{i=0}^{N} a_{i} z^{i} \in \mathbb{F}[z]$ with the finite support sequence $a_{0}=(a(z))_{0}, a_{1}=(a(z))_{1}, \ldots, a_{N}=(a(z))_{N}$ formed by its coefficients, and also use the notation $a(\ell)$ to denote $a_{\ell}=(a(z))_{\ell}$. The same applies for vectors with components in $\mathbb{F}[z]$.

A state-space system

$$
\left\{\begin{array}{c}
x(\ell+1)=A x(\ell)+B u(\ell) \\
v(\ell)=C x(\ell)+D u(\ell)
\end{array}, \ell \in \mathbb{N}_{0},\right.
$$

denoted by $(A, B, C, D)$, where $A \in \mathbb{F}^{\delta \times \delta}, B \in \mathbb{F}^{\delta \times k}, C \in \mathbb{F}^{n \times \delta}$ and $D \in \mathbb{F}^{n \times k}$, is said to be a state-space realization of the time-invariant $(n, k, \delta)$ convolutional code $\mathcal{C}$ if $\mathcal{C}$ is the set of codewords $v(z) \in \mathbb{F}^{n}[z]$ identified with the finite support output sequences $v$ corresponding to finite support input sequences $u$ (i.e., to information sequences $\left.u(z) \in \mathbb{F}^{k}[z]\right)$ and zero initial conditions, i.e., $x(0)=0$.

This definition implicitly assumes that $(A, B, C, D)$ is a minimal realization of $\mathcal{C}$, i.e., that $A$ has the minimal possible dimension [17]. 
State-space realizations of convolutional codes can be obtained as minimal statespace realizations of column reduced encoders. If $G(z) \in \mathbb{F}^{n \times k}[z]$ is an encoder of $\mathcal{C},(A, B, C, D)$ is a state-space realization of $G(z)$ if

$$
G(z)=C(I-A z)^{-1} B z+D .
$$

If $G(z)=\sum_{i \in \mathbb{N}_{0}} G_{i} z^{i}$, with $G_{i} \in \mathbb{F}^{n \times k}$, then

$$
G_{0}=D, G_{i}=C A^{i-1} B, i \geq 1 .
$$

Note that $G(z)$ admits many realizations. It is well-known that a state-space realization $(A, B, C, D)$ of $G(z)$ has minimal dimension among all the realizations of $G(z)$ if $(A, B)$ is controllable and $(A, C)$ is observable, i.e., the polynomial matrices $\left[z^{-1} I-A \mid B\right]$ and $\left[\begin{array}{c}z^{-1} I-A \\ C\end{array}\right]$ have, respectively, right and left polynomial inverses (in $z^{-1}$ ). The minimal dimension of a state-space realization of $G(z)$ is called the McMillan degree [9] of $G(z)$ and it is represented as $\mu(G)$.

The next proposition, adapted from $[6,7]$, provides a state-space realization for a given (not necessarily column reduced) encoder.

Proposition 1 Let $G(z) \in \mathbb{F}^{n \times k}[z]$ be a polynomial matrix with rank $k$ and column degrees $\nu_{1}, \ldots, \nu_{k}$. Consider $\bar{\delta}=\sum_{i=1}^{k} \nu_{i}$. Let $G(z)$ have columns $g_{i}(z)=\sum_{\ell=0}^{\nu_{i}} g_{\ell, i} z^{\ell}$, $i=1, \ldots, k$ where $g_{\ell, i} \in \mathbb{F}^{n}$. For $i=1, \ldots, k$ define the matrices

$$
A_{i}=\left[\begin{array}{rrrr}
0 & \cdots & \cdots & 0 \\
1 & & & \vdots \\
\ddots & & \vdots \\
& 1 & 0
\end{array}\right] \in \mathbb{F}^{\nu_{i} \times \nu_{i}}, B_{i}=\left[\begin{array}{c}
1 \\
0 \\
\vdots \\
0
\end{array}\right] \in \mathbb{F}^{\nu_{i}}, C_{i}=\left[g_{1, i} \cdots g_{\nu_{i}, i}\right] \in \mathbb{F}^{n \times \nu_{i}} .
$$

Then a state-space realization of $G$ is given by the matrix quadruple $(A, B, C, D) \in$ $\mathbb{F}^{\bar{\delta} \times \bar{\delta}} \times \mathbb{F}^{\bar{\delta} \times k} \times \mathbb{F}^{n \times \bar{\delta}} \times \mathbb{F}^{n \times k}$ where

$$
A=\left[\begin{array}{lll}
A_{1} & & \\
& \ddots & \\
& & A_{k}
\end{array}\right], B=\left[\begin{array}{lll}
B_{1} & & \\
& \ddots & \\
& & B_{k}
\end{array}\right], C=\left[\begin{array}{lll}
C_{1} & \cdots & C_{k}
\end{array}\right], D=\left[\begin{array}{lll}
g_{0,1} & \cdots & g_{0, k}
\end{array}\right]=G(0) .
$$

In the case where $\nu_{i}=0$ the ith block is missing and in $B$ a zero column occurs.

In this realization $(A, B)$ is controllable and if $G(z)$ is a column reduced encoder, $(A, C)$ is observable. Thus, the McMillan degree of a column reduced encoder is equal to the sum of its column degrees. 
3 State-space realizations of periodic convolutional codes

Definition 3 Let $\Sigma_{t}=\left(A_{t}, B_{t}, C_{t}, D_{t}\right), t=0,1$, be two state-space systems with the same dimension. We define a periodic state-space system $\Sigma_{p}$ as

$$
\left\{\begin{array}{rl}
x(\ell+1) & =A(\ell) x(\ell)+B(\ell) u(\ell) \\
v(\ell) & =C(\ell) x(\ell)+D(\ell) u(\ell)
\end{array}, \ell \in \mathbb{N}_{0}\right.
$$

where $A(\cdot), B(\cdot), C(\cdot), D(\cdot)$ are periodic functions with period 2 , such that for $j \in \mathbb{N}_{0}$

$$
(A(2 j), B(2 j), C(2 j), D(2 j))=\left(A_{0}, B_{0}, C_{0}, D_{0}\right)
$$

and

$$
(A(2 j+1), B(2 j+1), C(2 j+1), D(2 j+1))=\left(A_{1}, B_{1}, C_{1}, D_{1}\right) .
$$

The dimension of $\Sigma_{p}$ is defined as the dimension of the state vector $x$. In this case we say that $\Sigma_{p}$ is obtained from $\Sigma_{0}$ and $\Sigma_{1}$.

Moreover, $\Sigma_{p}$ is a realization of a periodic encoding map $\phi_{G^{0} G^{1}}$ if the output of $\Sigma_{p}$ that corresponds to an input $u(z)$ is equal to $\phi_{G^{0} G^{1}}(u(z))$, for all $u(z) \in \mathbb{F}^{k}[z]$.

Let $\Sigma_{0}$ and $\Sigma_{1}$ be two state-space realizations (of the same dimension) of two encoders $G^{0}(z)$ and $G^{1}(z)$. In the following example we show that the 2-periodic system $\Sigma_{p}$ obtained from $\Sigma_{0}$ and $\Sigma_{1}$ is not, in general, a state-space realization of $\phi_{G^{0} G^{1}}$ (see also [1]).

Example 2 Consider the two convolutional codes over $\mathbb{F}_{2}$ with encoders

$$
G^{0}(z)=G_{0}^{0}+G_{1}^{0} z+G_{2}^{0} z^{2}=\left[\begin{array}{ccc}
1+z^{2} & 1 & 0 \\
z^{2} & 1+z & 1 \\
1+z & 1 & 1 \\
1 & 1 & 1+z
\end{array}\right]
$$

and

$$
G^{1}(z)=G_{0}^{1}+G_{1}^{1} z+G_{2}^{1} z^{2}=\left[\begin{array}{ccc}
1+z & 1 & 0 \\
1+z^{2} & 1+z & 1 \\
1 & 1+z^{2} & 1 \\
0 & 1 & 1
\end{array}\right]
$$

Realizing $G^{0}(z)$ as in Proposition 1 we obtain the state-space realization $\Sigma_{0}=$ $(A(0), B(0), C(0), D(0))$ with

$$
A(0)=\left[\begin{array}{llll}
0 & 0 & 0 & 0 \\
1 & 0 & 0 & 0 \\
0 & 0 & 0 & 0 \\
0 & 0 & 0 & 0
\end{array}\right] \quad B(0)=\left[\begin{array}{lll}
1 & 0 & 0 \\
0 & 0 & 0 \\
0 & 1 & 0 \\
0 & 0 & 1
\end{array}\right] \quad C(0)=\left[\begin{array}{llll}
0 & 1 & 0 & 0 \\
0 & 1 & 1 & 0 \\
1 & 0 & 0 & 0 \\
0 & 0 & 0 & 1
\end{array}\right] \quad D(0)=\left[\begin{array}{lll}
1 & 1 & 0 \\
0 & 1 & 1 \\
1 & 1 & 1 \\
1 & 1 & 1
\end{array}\right]
$$

Proceeding the same way, we obtain a state-space realization of $G^{1}(z), \Sigma_{1}=$ $(A(1), B(1), C(1), D(1))$, with 


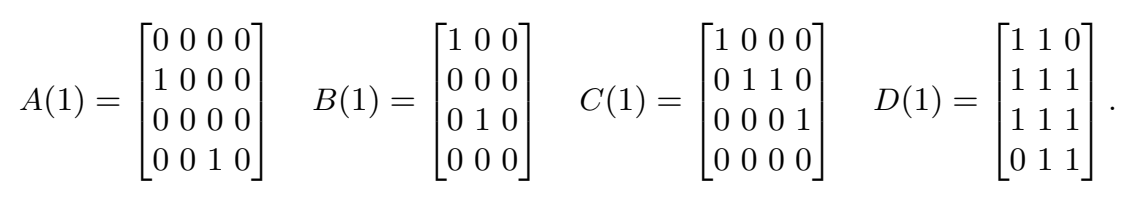

Let us consider $u(z)=u_{0}+u_{1} z$ with $u_{0}=\left[\begin{array}{l}0 \\ 0 \\ 1\end{array}\right]$ and $u_{1}=\left[\begin{array}{l}0 \\ 0 \\ 0\end{array}\right]$. From Definition 2 it follows that

$$
v_{1}=G_{0}^{1} u_{1}+G_{1}^{1} u_{0}=\left[\begin{array}{lll}
1 & 1 & 0 \\
1 & 1 & 1 \\
1 & 1 & 1 \\
0 & 1 & 1
\end{array}\right] u_{1}+\left[\begin{array}{lll}
1 & 0 & 0 \\
0 & 1 & 0 \\
0 & 0 & 0 \\
0 & 0 & 0
\end{array}\right] u_{0}=\left[\begin{array}{l}
0 \\
0 \\
0 \\
0
\end{array}\right]
$$

while from (5)

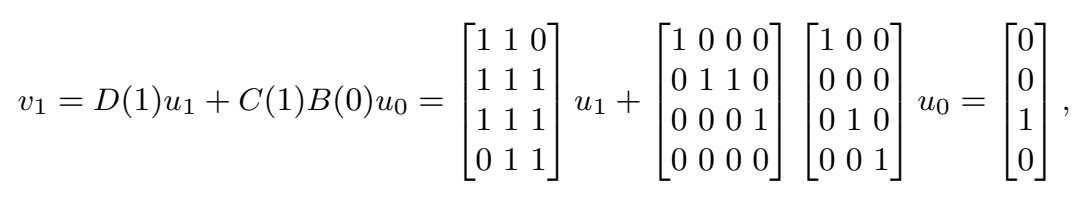

i.e., the output of the periodic state-space system $\Sigma_{p}$ obtained from $\Sigma_{0}$ and $\Sigma_{1}$ corresponding to $u(z)=u_{0}+u_{1} z$ is different from $\phi_{G^{0} G^{1}}(u(z))$.

In the next theorem we provide a sufficient condition for a periodic state-space system to be a realization of a periodic encoding map .

Theorem 1 Consider two encoders $G^{0}(z) \in \mathbb{F}^{n \times k}[z]$ and $G^{1}(z) \in \mathbb{F}^{n \times k}[z]$ with the same column degrees and let $\Sigma_{i}$ be the realizations of $G^{i}(z), i=0,1$ obtained by Proposition 1. Then, the periodic state-space system $\Sigma_{p}$ obtained from $\Sigma_{0}$ and $\Sigma_{1}$ is a realization of the periodic encoding map $\phi_{G^{0}} G^{1}$.

Proof Let us denote by $\Sigma_{0}=\left(A_{0}, B_{0}, C_{0}, D_{0}\right)$ and by $\Sigma_{1}=\left(A_{1}, B_{1}, C_{1}, D_{1}\right)$ the state-space realizations of $G^{0}(z)$ and $G^{1}(z)$ as in Proposition 1. Since the structure of the matrices $A_{0}, A_{1}, B_{0}$ and $B_{1}$ depends only of the column degrees of $G^{0}(z)$ and $G^{1}(z)$, we have that $A_{0}=A_{1}$ and $B_{0}=B_{1}$. Then the 2-periodic state-space system obtained from $\Sigma_{0}$ and $\Sigma_{1}$ has updating equations

$$
\left\{\begin{array}{rl}
x(\ell+1) & =A x(\ell)+B u(\ell) \\
v(\ell) & =C_{\ell-2\left\lfloor\frac{\ell}{2}\right\rfloor} x(\ell)+D_{\ell-2\left\lfloor\frac{\ell}{2}\right\rfloor} u(\ell)
\end{array}, \ell \in \mathbb{N}_{0},\right.
$$

where $A=A_{0}=A_{1}$ and $B=B_{0}=B_{1}$. Therefore, it follows from (4) that for any $u(z) \in \mathbb{F}^{k}[z]$,

$$
v(\ell)=D_{0} u(\ell)+\sum_{i=1}^{\ell} C_{0} A^{i-1} B u(\ell-i)=\left(G^{0}(z) u(z)\right)_{\ell}, \text { for } \ell=2 j
$$


and

$$
v(\ell)=D_{1} u(\ell)+\sum_{i=1}^{\ell} C_{1} A^{i-1} B u(\ell-i)=\left(G^{1}(z) u(z)\right)_{\ell}, \text { for } \ell=2 j+1,
$$

for $j \in \mathbb{N}_{0}$, i.e., $\Sigma_{p}$ is a 2 -periodic realization of $\phi_{G^{0} G^{1}}$.

In case $G^{0}(z)$ and $G^{1}(z)$ have different column degrees the following procedure can be applied in order to obtain a 2-periodic state-space realization of the periodic encoding map from state-space realizations of $G^{0}(z)$ and $G^{1}(z)$ :

1. Let $\nu_{i}$ be the maximum degree of the $i$-th columns of $G^{0}(z)$ and $G^{1}(z), i=$ $1, \ldots, k$;

2. Realize $G^{0}(z)$ and $G^{1}(z)$ as in Proposition 1 considering the columns of $G^{j}(z)$ as $g_{i}^{j}(z)=\sum_{\ell=0}^{\nu_{i}} g_{\ell, i}^{j} z^{\ell}, i=1, \ldots, k$, where some of the coefficients of higher degree may be zero.

Using this and the same line of arguments as in the proof of Theorem 1 the following theorem is immediate.

Theorem 2 Let $G^{0}(z), G^{1}(z) \in \mathbb{F}^{n \times k}[z]$ be two encoders with state-space realizations $\Sigma_{0}$ and $\Sigma_{1}$, respectively, obtained from the procedure above. Then the 2-periodic system obtained from $\Sigma_{0}$ and $\Sigma_{1}$ is a state-space realization of the periodic encoding map $\phi_{G^{0} G^{1}}$.

Example 3 Consider again the encoders of Example 2

$$
G^{0}(z)=G_{0}^{0}+G_{1}^{0} z+G_{2}^{0} z^{2}=\left[\begin{array}{ccc}
1+z^{2} & 1 & 0 \\
z^{2} & 1+z & 1 \\
1+z & 1 & 1 \\
1 & 1 & 1+z
\end{array}\right]
$$

and

$$
G^{1}(z)=G_{0}^{1}+G_{1}^{1} z+G_{2}^{1} z^{2}=\left[\begin{array}{ccc}
1+z & 1 & 0 \\
1+z^{2} & 1+z & 1 \\
1 & 1+z^{2} & 1 \\
0 & 1 & 1
\end{array}\right] .
$$

Let $\nu_{1}=2, \nu_{2}=2, \nu_{3}=1$ be the maximum degrees of the first, second and third columns, respectively, of $G^{0}(z)$ and $G^{1}(z)$. The state-space realizations $\Sigma_{0}=$ $(A, B, C(0), D(0))$ and $\Sigma_{1}=(A, B, C(1), D(1))$ of $G^{0}(z)$ and $G^{1}(z)$, respectively, obtained from the procedure above, are such that

$$
\begin{aligned}
& A=\left[\begin{array}{lllll}
0 & 0 & 0 & 0 & 0 \\
1 & 0 & 0 & 0 & 0 \\
0 & 0 & 0 & 0 & 0 \\
0 & 0 & 1 & 0 & 0 \\
0 & 0 & 0 & 0 & 0
\end{array}\right] \quad B=\left[\begin{array}{lll}
1 & 0 & 0 \\
0 & 0 & 0 \\
0 & 1 & 0 \\
0 & 0 & 0 \\
0 & 0 & 1
\end{array}\right] \\
& C(0)=\left[\begin{array}{lllll}
0 & 1 & 0 & 0 & 0 \\
0 & 1 & 1 & 0 & 0 \\
1 & 0 & 0 & 0 & 0 \\
0 & 0 & 0 & 0 & 1
\end{array}\right] \quad D(0)=\left[\begin{array}{lll}
1 & 1 & 0 \\
0 & 1 & 1 \\
1 & 1 & 1 \\
1 & 1 & 1
\end{array}\right]
\end{aligned}
$$




$$
C(1)=\left[\begin{array}{lllll}
1 & 0 & 0 & 0 & 0 \\
0 & 1 & 1 & 0 & 0 \\
0 & 0 & 0 & 1 & 0 \\
0 & 0 & 0 & 0 & 0
\end{array}\right] \quad D(1)=\left[\begin{array}{lll}
1 & 1 & 0 \\
1 & 1 & 1 \\
1 & 1 & 1 \\
0 & 1 & 1
\end{array}\right]
$$

The 2-periodic system obtained from $\Sigma_{0}$ and $\Sigma_{1}$ is a state-space realization of $\phi_{G^{0} G^{1}}$.

Assume that $\Sigma(\cdot)=(A(\cdot), B(\cdot), C(\cdot), D(\cdot))$ is a $\delta$-dimensional state-space realization of a periodic code $\mathcal{C}_{p}$, as presented below:

$$
\left\{\begin{array}{rl}
x(\ell+1) & =A(\ell) x(\ell)+B(\ell) u(\ell) \\
v(\ell) & =C(\ell) x(\ell)+D(\ell) u(\ell)
\end{array}, \ell \in \mathbb{N}_{0}\right.
$$

where $(A(\cdot), B(\cdot), C(\cdot), D(\cdot)) \in \mathbb{F}^{\delta \times \delta} \times \mathbb{F}^{\delta \times k} \times \mathbb{F}^{n \times \delta} \times \mathbb{F}^{n \times k}$ are periodic functions with period 2. Letting $w(\ell)=x(2 \ell)$ and $u^{L}(z)$ and $v^{L}(z)$ be defined as in (2), we obtain the following time-invariant $\delta$-dimensional state-space realization $\Sigma^{L}=$ $\left(A^{L}, B^{L}, C^{L}, D^{L}\right)$ for the lifted code $\mathcal{C}^{L}$ :

$$
\left\{\begin{array}{c}
w(\ell+1)=A^{L} w(\ell)+B^{L} u^{L}(\ell) \\
v^{L}(\ell)=C^{L} w(\ell)+D^{L} u^{L}(\ell)
\end{array}\right.
$$

with

$$
\begin{array}{rlrl}
A^{L} & =A(1) A(0) & B^{L} & =\left[\begin{array}{cc}
A(1) B(0) & B(1)
\end{array}\right] \\
C^{L} & =\left[\begin{array}{c}
C(0) \\
C(1) A(0)
\end{array}\right] & D^{L}=\left[\begin{array}{cc}
D(0) & 0 \\
C(1) B(0) & D(1)
\end{array}\right] .
\end{array}
$$

Thus, given a periodic realization $\Sigma(\cdot)$ for $\mathcal{C}_{p}$ there always exist an invariant realization $\Sigma^{L}$ for $\mathcal{C}^{L}$ with the same dimension as $\Sigma(\cdot)$. This holds, in particular, when $\Sigma(\cdot)$ is minimal; however the corresponding realization $\Sigma^{L}$ (with the same dimension) is not necessarily a minimal one. Therefore the minimal dimension of the realizations of $\mathcal{C}_{p}$ is greater or equal than the dimension of the (minimal) realizations of the corresponding lifted code $\mathcal{C}^{L}$. As mentioned earlier, this latter is equal to the McMillan degree of any column reduced encoder of $\mathcal{C}^{L}$.

In this context, it is natural to investigate when the two aforementioned minimal dimensions coincide.

Let us then assume that $\mathcal{C}_{p}$ is the periodic code obtained by two column reduced encoders $G^{0}(z), G^{1}(z) \in \mathbb{F}^{n \times k}[z]$ with the same column degrees, i.e., $\mathcal{C}_{p}=i m \phi_{G^{0} G^{1}}$ and that $\mathcal{C}^{L}$ is the lifted version of $\mathcal{C}_{p}$ with encoder $G^{L}(z) \in \mathbb{F}^{2 n \times 2 k}[z]$ as defined in (3). Moreover, let us consider state-space representations $\Sigma_{0}$ and $\Sigma_{1}$ of $G^{0}(z)$ and $G^{1}(z)$, respectively, as in Proposition 1, the 2-periodic system $\Sigma_{p}$ obtained from $\Sigma_{0}$ and $\Sigma_{1}$ and the corresponding state-space representation $\Sigma^{L}$ of $G^{L}(z)$ as defined in (6).

Let $\nu_{1}, \ldots, \nu_{k}$ be the column degrees of $G^{0}(z)$ and $G^{1}(z)$ and $g_{i}^{0}(z)$ and $g_{i}^{1}(z)$ be the $i$-th column of $G^{0}(z)$ and $G^{1}(z)$, respectively.

Write

$$
\left[\begin{array}{c}
G^{0}(z) \\
z^{-1} G^{1}(z)
\end{array}\right]=\left[\begin{array}{l}
G_{0}^{L_{0}}\left(z^{2}\right) \\
G_{1}^{L_{0}}\left(z^{2}\right)
\end{array}\right]+z^{-1}\left[\begin{array}{l}
G_{0}^{L_{1}}\left(z^{2}\right) \\
G_{1}^{L_{1}}\left(z^{2}\right)
\end{array}\right]
$$


where $G_{0}^{L_{0}}\left(z^{2}\right), G_{1}^{L_{0}}\left(z^{2}\right), G_{0}^{L_{1}}\left(z^{2}\right), G_{1}^{L_{1}}\left(z^{2}\right) \in \mathbb{F}^{n \times k}[z]$.

If $\nu_{i}=2 a$ is even, then the $i$-th column of $\left[\begin{array}{c}G_{0}^{L_{0}}\left(z^{2}\right) \\ G_{1}^{L_{0}}\left(z^{2}\right)\end{array}\right]$ has degree $2 a$ and leading column coefficient matrix $\left[\begin{array}{c}\left(g_{i}^{0}\right)_{\mathrm{hc}} \\ 0\end{array}\right]$ and the $i$-th column of $\left[\begin{array}{c}G_{0}^{L_{1}}\left(z^{2}\right) \\ G_{1}^{L_{1}}\left(z^{2}\right)\end{array}\right]$ has also degree $2 a$ and leading column coefficient matrix $\left[\begin{array}{c}* \\ \left(g_{i}^{1}\right)_{\mathrm{hc}}\end{array}\right]$.

On the other hand, if $\nu_{i}=2 b+1$ is odd, then the $i$-th column of $\left[\begin{array}{l}G_{0}^{L_{1}}\left(z^{2}\right) \\ G_{1}^{L_{1}}\left(z^{2}\right)\end{array}\right]$ has degree $2 b+2$ and leading column coefficient matrix $\left[\begin{array}{c}\left(g_{i}^{0}\right)_{\mathrm{hc}} \\ 0\end{array}\right]$ and the $i$-th column degree of $\left[\begin{array}{l}G_{0}^{L_{0}}\left(z^{2}\right) \\ G_{1}^{L_{0}}\left(z^{2}\right)\end{array}\right]$ is $2 b$ and corresponding leading column coefficient matrix $\left[\begin{array}{c}* \\ \left(g_{i}^{1}\right)_{\mathrm{hc}}\end{array}\right]$.

Since $\left(G^{0}\right)_{\mathrm{hc}}$ and $\left(G^{1}\right)_{\mathrm{hc}}$ are full column rank, it follows that also the leading column coefficient matrix of

$$
G^{L}(z)=\left[\begin{array}{ll}
G_{0}^{L_{0}}(z) & G_{0}^{L_{1}}(z) \\
G_{1}^{L_{0}}(z) & G_{1}^{L_{1}}(z)
\end{array}\right]
$$

is full column rank and therefore $G^{L}(z)$ is column reduced. Moreover, the sum of the $i$-th column degrees of $\left[\begin{array}{l}G_{0}^{L_{0}}(z) \\ G_{1}^{L_{0}}(z)\end{array}\right]$ and $\left[\begin{array}{l}G_{0}^{L_{1}}(z) \\ G_{1}^{L_{1}}(z)\end{array}\right]$ is equal to $\nu_{i}$, which means that $G^{L}(z)$ is a column reduced encoder with sum of the column degrees equal to the sum of the column degrees of $G^{i}(z), i=0,1$. Then $\mu\left(G^{L}\right)=\mu\left(G^{0}\right)=\mu\left(G^{1}\right)$, and the next theorem follows.

Theorem 3 Let $G^{0}(z), G^{1}(z) \in \mathbb{F}^{n \times k}[z]$ be two column reduced encoders with the same column degrees and let $\Sigma_{i}$ be the state-space realizations of $G^{0}(z)$ and $G^{1}(z)$, respectively, as in Proposition 1. Then the corresponding 2-periodic state-space realization obtained from $\Sigma_{0}$ and $\Sigma_{1}$ is minimal.

\section{Conclusions}

In this paper we have studied the relation between periodic convolutional codes and periodic input-state-output representations. We showed that this connection is not as straightforward as it seems. In fact, input-state-output realizations of each one of two reduced encoders does not necessarily yield a periodic input-state-output realization of the associated periodic code. First we presented a direct method to obtain an input-state-output realization under certain conditions. Then we gave a procedure to still obtain such a realization even if these conditions are not satisfied. Conditions for these realizations to be minimal were also investigated. 


\section{References}

1. Aleixo, J.C., Rocha, P.: Roesser model representation of 2 d periodic behaviors: the $(2,2)-$ periodic siso case. In: 2017 10th International Workshop on Multidimensional (nD) Systems (nDS), pp. 1-6 (2017). DOI 10.1109/NDS.2017.8070617

2. Aleixo, J.C., Rocha, P., Willems, J.C.: State space representation of siso periodic behaviors. In: 2011 50th IEEE Conference on Decision and Control and European Control Conference, pp. 1545-1550 (2011). DOI 10.1109/CDC.2011.6160552

3. Bocharova, I.E., Kudryashov, B.D.: Rational rate punctured convolutional codes for softdecision viterbi decoding. IEEE Transactions on Information Theory 43(4), 1305-1313 (1997). DOI 10.1109/18.605600

4. Costello, D.J.: Free distance bounds for convolutional codes. IEEE Transactions on Information Theory 20(3), 356-365 (1974). DOI 10.1109/TIT.1974.1055223

5. Fekri, F., Sartipi, M., Mersereau, R.M., Schafer, R.W.: Convolutional codes using finitefield wavelets: time-varying codes and more. IEEE Transactions on Signal Processing 53(5), 1881-1896 (2005). DOI 10.1109/TSP.2005.845484

6. Fornasini, E., Pinto, R.: Matrix fraction descriptions in convolutional

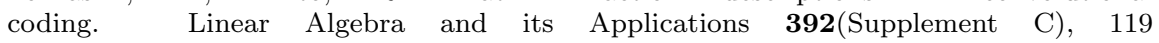
- 158 (2004). $\quad$ DOI https://doi.org/10.1016/j.laa.2004.06.007. URL http://www.sciencedirect.com/science/article/pii/S0024379504002836

7. Gluesing-Luerssen, H., Schneider, G.: State space realizations and monomial equivalence for convolutional codes. Linear Algebra and its Applications 425(2), 518 - 533 (2007). DOI https://doi.org/10.1016/j.laa.2007.03.004. URL http://www.sciencedirect.com/science/article/pii/S002437950700122X. Special Issue in honor of Paul Fuhrmann

8. Johannesson, R., Zigangirov, K.S.: Fundamentals of Convolutional Coding. IEEE Press, New York (1999)

9. Kailath, T.: Linear systems. Prentice Hall information and system sciences series. PrenticeHall, Englewood Cliffs (1980)

10. Kuijper, M., Polderman, J.: Reed-Solomon list decoding from a system theoretic perspective. IEEE Trans. Inf. Th. IT-50, 259-271 (2004)

11. Kuijper, M., Willems, J.C.: A behavioral framework for periodically time-varying systems. In: Proceedings of the 36th IEEE Conference on Decision and Control, vol. 3, pp. 20132016 vol.3 (1997). DOI 10.1109/CDC.1997.657060

12. Lee, P.J.: There are many good periodically time-varying convolutional codes. IEEE Transactions on Information Theory 35(2), 460-463 (1989). DOI 10.1109/18.32142

13. Mooser, M.: Some periodic convolutional codes better than any fixed code (corresp.). IEEE Transactions on Information Theory 29(5), 750-751 (1983). DOI 10.1109/TIT.1983.1056727

14. Napp, D., Perea, C., Pinto, R.: Input-state-output representations and constructions of finite support 2D convolutional codes. Advances in Mathematics of Communications 4(4), $533-545(2010)$

15. Palazzo, R.: A time-varying convolutional encoder better than the best time-invariant encoder. IEEE Transactions on Information Theory 39(3), 1109-1110 (1993). DOI $10.1109 / 18.256526$

16. Rosenthal, J.: Connections between linear systems and convolutional codes. In: B. Marcus, J. Rosenthal (eds.) Codes, Systems and Graphical Models, IMA Vol. 123, pp. 39-66. Springer-Verlag (2001)

17. Rosenthal, J., York, E.V.: BCH convolutional codes. IEEE Trans. Automat. Control 45(6), 1833-1844 (1999)

18. Truhachev, D., Zigangirov, K.S., Costello, D.J.: Distance bounds for periodically timevarying and tail-biting ldpc convolutional codes. IEEE Transactions on Information Theory 56(9), 4301-4308 (2010). DOI 10.1109/TIT.2010.2053873

19. Viterbi, A.: Convolutional codes and their performance in communication systems. IEEE Transactions on Communication Technology 19(5), 751-772 (1971). DOI 10.1109/TCOM.1971.1090700 